Indexaciones: Repositorio de Revistas UCR, DIALNET, Latindex, REDALYC Directorio y recolector de recursos digitales del Ministerio de Cultura de España, Directory of Open Access Journals. Diálogos Revista Electrónica de Historia ISSN 1409- 469X. Número especial 2008. Dirección web: http://historia.fcs.ucr.ac.cr/dialogos.htm

\title{
Reseña Histórica de la Prueba de Aptitud Académica (PAA-U.C.R.)
}

\author{
Dra. Aida $\mathrm{M}^{\mathrm{a}}$ Mainieri Hidalgo \\ Psicóloga Investigadora \\ Instituto de Investigaciones Psicológicas \\ Programa Permanente Prueba de Aptitud Académica \\ Universidad de Costa Rica \\ Telfs.: 2207-5725 y 2271-3222 \\ Fax.: 2271-3222 \\ E-mail: aida.mainieri@ucr.ac.cr \\ amainier@racsa.co.cr
}


"Para escribir una historia exitosa es necesario contar con distintos elementos. Algunos de ellos son: una gran idea, empeño, filosofía de trabajo, misión, actitud vanguardista y seguir el pulso del cambio mundial” (Estrada, R.: 2008, p.74). Esta es la historia que construyeron en el pasado los creadores de la PAA-UCR, se ha reconstruido a lo largo de casi 50 años y ahora tenemos en nuestras manos.

\section{INTRODUCCIÓN:}

Este estudio, se da con el objeto de reconstruir históricamente los fundamentos teóricos, metodológicos, técnicos y prácticos que han sustentado la toma de decisiones en torno al diseño, aplicación, utilización y evaluación de la Prueba de Aptitud Académica de la Universidad de Costa Rica, desde sus inicios hasta la actualidad. En su metodología, se parte de un enfoque hermenéutico, de manera que se atienden los emergentes relevantes; se lleva a cabo por medio de un análisis bibliográfico - documental y recurriendo luego a una metodología participativa con lo que se parte de entrevistas en profundidad a sus constructores, diseñadores y a expertos.

La Prueba de Aptitud Académica inicia en 1957, con el nombramiento del Comité de Evaluación para la construcción de la misma y en el que figura, desde entonces, la persona del Dr. Gonzalo Adis Castro, su creador y fiel desarrollador. Fue implementada y aplicada por primera vez y en forma generalizada en 1960, año mismo en el que, dicho comité, pasa a constituirse en el Instituto de Investigaciones Psicológicas con el objetivo primordial de darle seguimiento. Podemos decir entonces que la PAA-UCR cuenta ya con una trayectoria de 48 años y celebraría sus 50 años en el 2010, año mismo en que la UCR celebrará su 70 aniversario.

Al tener por objeto la valoración de las aptitudes académicas de los aspirantes a ingreso a la UCR, esta prueba se ve afectada por el proceso psicogenético de la población de estudiantes, respondiendo al contexto sociocultural y educativo que hoy en día se enmarcan en la revolución científico-tecnológica y de la sociedad del conocimiento; esto si se la analiza desde la epistemología genética de Piaget, en que la psicogénesis comprende tanto el nivel filogenético, o evolución psíquica del ser humano a través de su desarrollo como especie, como el ontogenético, o evolución psíquica del individuo humano a partir de su gestación biológica y a lo largo de su vida. Este hecho también se sustenta en el principio de la diferenciación cultural o Ley de Ferguson (1956) de la diferenciación cultural, "los factores culturales prescriben qué debemos aprender y a qué edad; consecuentemente, diferentes ambientes culturales conducen al desarrollo 
de diferentes patrones de aptitudes” (Espinosa, M.: 1997, p. 121). De hecho, los análisis de calidad técnica (dificultad y discriminación), a los que se someten los ítems que conforman dicho instrumento, dan como resultado una movilidad constante; así observamos cómo algunos de estos reactivos resultaron óptimos en el pasado y con el tiempo se van descartando, requiriendo reemplazo.

A la PAA, la podemos catalogar como un Test de selección de alto impacto, dado que fue creada y ha sido utilizada para elegir a los mejores candidatos a cursar carreras en la UCR, es decir, para una toma de decisiones de altas consecuencias para quienes la realizan, como requisito de ingreso a la UCR y para los objetivos y fines de la Educación Superior y de la sociedad a la que sirve.

El ingreso a la Universidad de Costa Rica, la deserción y en consecuencia la creación del Comité de Evaluación y la Prueba de Aptitud Académica son eventos que se encuentran interrelacionados, de tal manera que no podemos referirnos a cada uno de ellos por separado sin perder la esencia; asimismo, es a partir de ello que se crea la Escuela de Ciencias del Hombre, de la que surge la Escuela de Psicología, y en ese mismo orden cronológico se sientan las bases con las que nace la Psicología en Costa Rica.

A partir de 1941, cuando se inicia la reforma de la Universidad, un año después de su fundación en 1940, y se da la integración progresiva de nuevas carreras, el ingreso de nuevos/as estudiantes era cada vez mayor. Eso, aunado a las consecuencias de una explosión demográfica sostenida, en la que se daban tasas de crecimiento, no de candidatos ni de aspirantes, sino de población de ingreso que pasaba directamente a la educación superior, y a la única universidad de entonces, la UCR, dio como consecuencia que la deserción aumentaran en forma continua y alarmante. Asimismo, se acrecentaba un mal logro de los programas educativos que se ofrecían, dada una población de estudiantes admitidos que sobrepasaba cada vez más las posibilidades de la capacidad instalada; con ello aumentaba el malestar del profesorado que se quejaba de no poder atender debidamente a los alumnos que sí serian los futuros profesionales en sus campos. Así se fue conformando un semillero del que posteriormente germina la PAA. Ella nace como respuesta a la necesidad manifestada por varias facultades de realizar una prueba, en la cual se deje al descubierto si el estudiante de nuevo ingreso posee la preparación suficiente para cursar estudios universitarios.

Según los datos recolectados, tal y como se evidencia más adelante, no es sino hasta once 
años después, en 1952, que el Rector, Don Rodrigo Facio, toma acciones sobre su preocupación e inquietud con respecto a la deserción desmedida de los y las estudiantes y especialmente la de los y las de primer ingreso. A partir de ese año es que algunas escuelas inician la aplicación de pruebas de ingreso; pruebas que inicialmente no eran de aptitudes sino que se trataba de diversos tests psicológicos que luego son sustituidas por pruebas de conocimientos. No es hasta el año 1960, después de un impás 3 años en el que no se efectuó prueba alguna, que se aplica por primera vez una PAA, como prueba de selección de los estudiantes a la UCR, esto en medio del nacimiento de la Psicología en el país.

Esta prueba fue tomada del "Scholastic Aptitute Test" (SAT); una prueba de aptitud académica que se ha venido desarrollando desde principios de siglo, cuando se inició este tipo de práctica de selección de estudiantes para la educación superior en los Estados Unidos y en su adopción ha participado el College Entrance Examination Board de Puerto Rico, hoy en día el College Board PRAL (Para América Latina).

Sus bases teóricas son muy amplias; las podemos encontrar en la psicometría, la estadística, las teorías acerca de la inteligencia, habilidades y aptitudes, así como en la epistemología y las teorías referentes al aprendizaje y a la cognición que se desprenden de los distintos paradigmas epistemológicos. También encontramos un sinnúmero de estudios acerca de problemas de rendimiento académico, estudios de predictividad del éxito académico en estudiantes universitarios en la UCR, en otras universidades estatales que trabajan con la misma población y, en otros países, todos ellos resultan de gran interés.

\section{PRIMEROS PASOS DELPROCESO DE SELECCIÓN DE ESTUDIANTES, GESTACIÓN DE LA PAA-UCR:}

1942: Haciendo un recorrido por la historia, encontramos que el 23 de diciembre de 1942 el Ing. Tinoco pone de manifiesto la necesidad de establecer exámenes previos de admisión a los estudiantes, argumentando que no todos los solicitantes tenían la preparación suficiente para realizar con éxito sus estudios universitarios. Ante esto el Lic. Edgar González aclara que no sería ilegal establecer este tipo de prueba, debido a que en la normativa interna de la Universidad no se establecía como requisito indispensable de ingreso, el bachillerato, por lo tanto se podrías considerar que cada Escuela Universitaria exigiera exámenes previos sobre sus materias más relevantes. El Sr. Rector consideró que lo más prudente era que la Facultad presentara al Consejo Universitario (CU) un plan concreto sobre el asunto. (Sesión 42.E07-08 del 23-12-42, Tomo: 01 Folio: 263) 
1948: Posteriormente, en octubre de 1948, el profesor Rafael Zúñiga presenta como moción la conveniencia de conocer en todos sus aspectos las condiciones de los estudiantes que ingresan a la Universidad, esto mediante una investigación apropiada. Por lo tanto, el CU acuerda: "Integrar una comisión que formule una serie de "tests” a la que serán sometidos todos los alumnos de los primeros años de la universidad” y se indica como posibles puntos a ser investigados: conocimientos básicos en materias como matemática, y aspectos personales fundamentales; los resultados obtenidos serían tabulados y analizados por cada escuela, y si la experiencia resultare satisfactoria se pensaría en la expansión a otros sectores. (Sesión 48.34-08 del 18-10-48, Tomo: 04 Folio: 457)

1949: El 21 de febrero de 1949, la prof. Gamboa explica que por razones de espacio “este año no será posible acomodar al numeroso grupo de estudiantes que suelen matricularse para el primer año en la Escuela de Pedagogía” y sugiere que se autorice la limitación de los estudiantes que asistan, sobre la base de exámenes de ingreso que la facultad podría hacer. (Sesión 49.08-21 del 21-02-49, Tomo: 05 Folio: 71)

1950: Un año más tarde, la Comisión de Planes de Estudio y Programas acuerda discutir ampliamente la petición realizada por la Facultad de Ingeniería de que se establezca un examen de admisión sobre tres materias fundamentales, para los jóvenes que deseen ingresar al primer año de dicha facultad (Sesión 50.048-17 del 23-01-50, Tomo: 05 Folio: 405). Posteriormente, en septiembre del mismo año, el Prof. Zúñiga presenta una iniciativa para establecer exámenes de admisión al primer año de las diversas Escuelas Universitarias, con el fin de elevar el nivel cultural del estudiantado, y sugiere sea implantado para el curso lectivo de 1951. Ante esta iniciativa el CU. acuerda solicitar la opinión a cada facultad. .(Sesión 50.49-09 del 25-09-50, Tomo: 06 Folio: 160).

1951: El 08 de enero de 1951, la Facultad de Derecho manifiesta su acuerdo con la propuesta del profesor Zúñiga de que se establezcan exámenes previos de admisión. (Sesión 51.02-10 del 08-01-51, Tomo: 06 Folio: 259). El 10 de diciembre se consulta a los decanos presentes al respecto, contestando afirmativamente los de Ingeniería, Cirugía Dental, Pedagogía, Derecho y Ciencias Económicas, a la vez se presenta el reglamento de exámenes de admisión que se pensaba aplicar para el año siguiente (Sesión 51.51-17 del 10-12-51, Tomo: 07 Folio: 91). Por consiguiente, se nombró una comisión integrada por los s Lics. Ortiz, Ruiz y Fallas para que 
estudiara el dicho reglamento que ya había sido aprobado por la Facultad de Ciencias Económicas y Sociales y rindiera un informe. (Sesión 51.51-20 del 10-12-51, Tomo: 07 Folio: 91)

El 18 de diciembre de 1951, se lee y aprueba el dictamen expuesto por la Comisión de Planes de Estudio referente al proyecto de exámenes de admisión, dicho dictamen se manifiesta a favor de una prueba en cuatro ramas principales: Humanidades, Ciencias Físicas, Biológicas y Sociales. Con el adendum de que "tiene el inconveniente de no poner el énfasis de la prueba en aquellas áreas o disciplinas que le van a servir al joven para la formación profesional que garantiza la facultad” y que el número de preguntas sugeridas “puede llevar a contestaciones que no revelarían juicios ni formas de pensamiento, sino el dominio de una dispersa información enciclopédica” se sugirió como base de la prueba los aspectos siguientes: Matemáticas, Castellano, Ciencias Sociales y un Idioma Extranjero.

Por otra parte, las preguntas estarían distribuidas de modo tal, que el mayor número de éstas correspondiera a conocimientos y aptitudes vocacionales relacionadas con la formación profesional ofrecida por la facultad, ya que dicha prueba "no debe ser una repetición del examen de bachillerato o algo por el estilo, sino una oportunidad que tendrán los jóvenes para revelar sus aptitudes, intereses y conocimientos” relacionados con la formación que se adquiere o desarrolla cada escuela.

La Comisión también propició como complemento a la prueba "hacer entrevistas con el objeto de conocer un poco más a fondo los ideales, aspiraciones, actitudes, opiniones, dudas, etc. de los (as) muchachos (as)". A su vez recomendó "la creación de una oficina permanente encargada de investigar los problemas de orden pedagógico - psicológico, y en general de orientación vocacional de la UCR. Por otra parte se dispuso publicar un aviso dando cuenta de los nuevos requerimientos de ingreso a la Escuela de Ciencias Económicas y Sociales para el siguiente año e informar que las Facultades de Derecho, Ingeniería y Odontología exigirían también pruebas de admisión. (Sesión 51.E53- 05 del 18-12-51, Tomo: 07 Folio: 103).

1952: A propósito de la prueba, en enero de 1952, el profesor Monge "sugiere que la Universidad no cobre suma alguna a los alumnos por esos exámenes”(Sesión 52.01-31 del 0701-52, Tomo: 07 Folio: 127), por su parte la Dra. Gamboa sugiere la conveniencia de realizar una publicación con el fin de aclarar las razones para exigir requisitos de admisión, y referirse a la posible creación de una oficina de orientación estudiantil; a la vez, ella deseaba brindar 
aclaraciones acerca de publicaciones inexactas al respecto (Sesión 52.01- 03 del 07-01-52, Tomo: 07 Folio: 127). También, la Facultad de Pedagogía presenta el proyecto correspondiente para las pruebas de admisión que exigirá.

El 14 de enero, de 1952, se lee una comunicación de la Facultad de Derecho que reitera su decisión de proceder a aplicar pruebas de admisión ese año e indica que dichas pruebas deben quedar bajo la reglamentación general a cargo de la Secretaría General (Sesión 52.01- 03 del 14-01-52, Tomo: 07 Folio: 133). Este mismo día, se mencionan diversos aspectos acerca de cómo debería funcionar la nueva oficina, sugiriendo el profesor Gámez un plan de inserción para estudiantes nuevos. (Sesión 52.02- 11 del 14-01-52, Tomo: 07 Folio: 133). Este plan fue aprobado el 28 de enero del mismo año.

Así las cosas, es en 1952 que la llamada Secretaria General fue organizada en tres grandes departamentos o áreas de trabajo: Proveeduría, Extensión Cultural y Bienestar Estudiantil. Esta iniciativa se da bajo la necesidad establecida por el Consejo Universitario de que una comisión para que planeara este Departamento, como respuesta a la necesidad sentida por los estudiantes. "Mueve a nuestra Universidad la justificada inquietud por organizar y poner en actividad este Departamento” (Gámez , 1952, Pág. 31). Este Departamento tenía también como labor, investigar lo que estaba sucediendo en otras universidades en el exterior para proceder a tomar medidas en lo interno, por lo que se fueron implementado nuevos programas. (Gámez, 1952, Pág. 31).

1953: Dentro de sus primeras acciones, al Dep. de Bienestar y Orientación le correspondía la aplicación del Cuestionario de Adaptación Escolar, esto con el fin de conocer los problemas que allí se plantean para proyectar los medios de hallarles solución.

\section{Con la colaboración del Dep de la U de Puerto Rico, se obtuvieron una cantidad de Test} traducidos, a partir de los cuales se formuló un plan de requisitos de ingreso a la UCR, el cual fue sometido al Consejo Universitario y aprobado por este, para ser aplicado a partir de 1954 en todas las facultades, aparte de los "Exámenes de Conocimientos Específicos Básicos" que cada Escuela aplicaba. Los requisitos eran: Examen Medico, Entrevista, Test de Inteligencia, Test de Aptitudes Vocacionales, Examen de Lenguaje.

La Escuela de Pedagogía realiza sus propias pruebas: Entrevista, Test de conocimientos básicos, Prueba de Castellano (lectura, dicción, ortografía y redacción), Test de Exploración Vocacional para el Magisterio (Formulas de la Universidad de Minnesota), (Gámez Solano, U.: 1953). 
1954: El objetivo del Departamento de Bienestar y Orientación es responder al interés que tiene la Universidad de "que los estudiantes asistan a las aulas, donde tengan las mejores oportunidades, experiencias y estímulos para su desarrollo intelectual, espiritual y social que los(as) capacite, no solo para incorporarse a la vida profesional, sino además para llenar en forma valiosa las funciones ciudadanas y contribuir eficazmente al proceso cultural del país. (Coronado, 1954, Pág. 162)

El psicólogo puertorriqueño Prof. Guillermo Velásquez Flores, que fue contratado temporalmente para el trabajo en orientación, lo que era un gran aporte, ya que se ofrecía por primera vez en el país, desde una institución educacional, servicios completos de orientación: personal, vocacional, y académica, empleando recursos de la Psicometría Científica. El Sr. Velásquez impartió un curso de Psicometría que fue aprovechado por un buen número de profesores y funcionarios. Se importaron algunos tests, se revisaron las traducciones y se seleccionaron las pruebas que se estimaron más aprovechables para los fines deseados; este año se tenían 19 tests en uso.

En esta época se muestra una profunda preocupación por la deserción de los estudiantes, que es percibida como alta, y para lo que se iniciaron algunos sondeos sobre la situación: "se entrevistó a 139 estudiantes que se retiraron ... esperamos arrojará mucha luz sobre diversos problemas relacionados con el gran volumen de deserciones, lo que preocupa a las autoridades universitarias" (Coronado, 1954, Pág. 171). El CU había acordado un porcentaje mínimo del $30 \%$ en la prueba, como requisito de ingreso.

Para el Señor Rector, Rodrigo Facio (Universidad de Costa Rica, 1954), estos exámenes no constituían un sistema definitivamente probado y comprobado para la Institución, sino que representaba una experiencia aplicada con fines más bien experimentales, apareciendo a ella asociados diferentes propósitos y preocupaciones acerca de cómo mejorar la selección del estudiantado, más adecuado aprovechamiento de los escasos recursos académicos y financieros, materiales y técnicas con los que la Universidad contaba, análisis de la preparación de los candidatos y orientación de los mismos. (Rodrigo Facio, Informe del Rectoría, 1954)

Personeros de la UCR, con el objeto de iniciar la orientación de los estudiantes antes de que ingresaran, visitaron 29 colegios de Segunda Enseñanza durante los meses de septiembre, octubre y noviembre (UCR Anales: 1954).

1955: En este año se continuaron las visitas a los colegios, esto como un servicio de orientación 
vocacional que consistía en: una prueba psicológica y una o más entrevistas que ayudara a los solicitantes a formular una decisión vocacional lo más acertada posible.

Para este año fue creado el Departamento de Registro, encargado de los requisitos y procedimientos de admisión. Siendo el profesor Carlos Monge quien redactó una guía con los requisitos de ingreso. Además, el Departamento de Orientación aplicó “Exámenes de Habilidad General” como las que se vienen mencionando, a todos los aspirantes a matricula, pruebas que respaldaban las conclusiones derivadas de los Exámenes de Conocimientos Básicos.

En diciembre del 54, el Sr. Velásquez Había terminado su contrato y regresó a Puerto Rico. (Coronado, M.: 1955) (Facio, Rodrigo: 1955).

1956: El Departamento de Bienestar y Orientación muestra preocupación por el incremento de los estudiantes, y la necesidad de que la Universidad proveyera de forma más organizada y sistemática los servicios de orientación, con el objeto de lograr que los estudiantes resolvieran sus problemas de formación general y profesional. "La población estudiantil universitaria ha ido creciendo año con año, y es lógico esperar que el mismo ritmo, un tanto más acelerado continúe en el futuro" (Padilla. 1954, pág.653) (UCR Anales: 1956).

\section{IMPLEMENTACIÓN DE LA PAA-UCR}

1957: A principios de 1957, el CU tomó la decisión de definir y establecer un sistema de admisión propiamente dicho, en previsión del hecho de que conforme aumentara el número de estudiantes que solicitaban ingreso a la Universidad, se irían presentando serias limitaciones de profesores, planta física y equipo en los laboratorios de enseñanza. (C.U. Política de Admisión. Antecedentes, 1991). Este sistema de admisiones debía: permitir, por una parte ajustar el número de alumnos a dichas limitaciones y por otra, disminuir el alto porcentaje de deserción, dar oportunidad a los estudiantes mejor preparados para los estudios superiores y ayudar a asegurar un alto nivel de rendimiento (C.U. Correspondencia octubre 1991).

EI III Congreso Universitario se ocupa de la problemática anterior, recomendando definir una "política de admisión que responda básicamente a capacidades intelectuales y conocimientos básicos” del estudiante; así el debía, además, ser diferenciado, tomando en cuenta las condiciones económicas, sociales, de procedencia, etc. de los aspirantes (C.U. Correspondencia octubre 1991). Las resoluciones de este congreso se materializan en el Estatuto Orgánico, en el que se define el requerimiento de un "Régimen de Admisión que se 


\section{sustentara en una Prueba de Aptitud Académica”; a su vez, desde ese momento el cupo} pasó a ser el parámetro para definir la nota mínima de ingreso a la Universidad (C.U. Correspondencia octubre 1991).

Para este año las pruebas de conocimientos básicos fueron suspendidas, excepto en la Escuela de Educación. El origen de esta decisión se fundamentó en la urgencia de que debía encontrarse, tanto en la reforma académica iniciada ese año como en la ampliación de facilidades materiales, el establecimiento de un primer año común, con cursos de Estudios Generales y actividades de tipo formativo. Por otra parte, se perfilaba la reforma del edificio de Ciencias y Letras con el objeto de adecuar las posibilidades físicas y académicas de la institución a esa necesidad.

Los estudiantes que aspiraran al paso a Escuelas Profesionales debían aprobar el primer año de Ciencias y Letras, que respondía a un sistema de cupo limitado, pero luego se cambio la directriz estipulando que se tenían que aprobar integralmente las asignaturas de primer año de Ciencias y Letras, todo con la intención de resolver el problema de exceso de estudiantes e introducir un sistema ordenado y lógico en los estudios.

Desde el 1 de marzo de este año, los Servicios de Orientación estuvieron bajo la Dirección del Dr. Gonzalo Adis Castro, quien, según el Sr. Rector Rodrigo Facio (UCR, 1957), el aporte dado es de gran calidad científica, "que nos permite esperar muy valiosas colaboraciones en el futuro, no solo en el campo del a orientación y la psicoterapia, sino además en la investigación y la docencia” (Coronado, 1958, Pág. 679) (Padilla, E. (1957).

1958: Durante este año no se aplicaron pruebas de conocimiento básicas par el ingreso a la Universidad, con excepción de la Facultad de Educación. Las razones de la regla y de la excepción fueron las mismas a las del año anterior. Tampoco se impusieron cuotas para el paso de la Escuela de Ciencias y Letras a las Escuelas Profesionales. Según los informes presentados por el Dr. Adis, existe una “desorientación vocacional” como causa de deserción universitaria; en este año se inician estudios de investigación a su cargo. (Coronado, M.: 1958).

Desde fines del 58, la Facultad de Ciencias y Letras estaba encargada del estudio de un “Sistema Racional de Selección” que pudiera ser aplicado en los años siguientes; así, se encomendó esta tarea a un Comité de Evaluación constituido por el Dr. Gonzalo Adis Castro; el Lic. Carlos Caamaño, Vicedecano de la Facultad de Ciencias y Letras y persona a cargo de la Coordinación del Comité; el Dr. Edgar González, Psicólogo Educacional; Lic. Rodrigo Umaña, 
Profesor de la Escuela de Estadística e Isaac Felipe Azofeifa, Filósofo, Poeta y Profesor de Estudios Generales; “a menudo también contábamos con la invaluable ayuda del Mr. Miguel Gómez, de la Escuela de Estadística, quien colaboraba con nosotros en lo relacionado con los análisis estadísticos que se realizaban", otra fiel colaboradora fue la Licda. Rosa María Nielsen (Adis C., G.: Inédito p. 6 y 7). “En cuanto a Isaac Felipe ... fuimos colegas de trabajo e hicimos juntos el primer exámen de admisión, él ayudó con la formulación de preguntas y yo ayudé con técnicas de construcción de pruebas; era la primera prueba, estaba Isaac Felipe y un estadístico, Rodrigo Umaña, colaboró también Edgar Gonzalez y alguien más” (Adis, G: entrev. 1).

El Comité resolvió que era necesario incluir una prueba de Aptitud General que diera un índice de la habilidad del individuo para manejar y aplicar conceptos abstractos, particularmente abstractos verbales. Así la prueba comprendió tres partes: las dos primeras abarcan dos distintos aspectos de manejo de vocabulario y la tercera compresión de lectura. (UCR Anales: 1959); “... algo que contribuyó a darle mayor visibilidad a la Psicología fue la construcción de la PAA o Examen de Admisión. Para su construcción el Comité de Evaluación siguió un modelo investigativo, conocido como “modelo empírico a posteriori” (Adis, Azofeifa y Umaña: 1960 p. 15-20) el que se ha continuado utilizando”. Este modelo versa con un “modelo empírico a priori”, en el que se debería establecer los procesos mentales que se van a evaluar en cada ítem previamente a su construcción, lo que no ha sido posible poner en práctica.

1959: Tampoco, se exigieron pruebas de conocimiento para el ingreso a la Universidad, con la excepción de las requeridas en la Sección de Primera Enseñanza de la Facultad de Educación, todo con miras a que en el siguiente año sí se aplicaran los “Exámenes Generales de Admisión, que incluían la nueva PAA-UCR.

Se comprende que el objetivo de aplicar dichas prueba se concentraba en seleccionar a los jóvenes con talento, disposición y/o voluntad para seguir los estudios superiores, frenar el acceso de jóvenes que carecieran de un perfil para rendir adecuadamente en la universidad, reducir la tasa de deserción motivada por los bajos rendimientos académicos y señalar el requerimiento de canalizar a esa población hacia otros programas educativos, en los que resultaran más exitosos. Sin embargo, algunas expresiones escritas que se encuentran en la documentación parecen desvirtuar estos objetivos y proyectan la imagen de la Prueba como un "filtro". Así encontramos por escrito: “con el objetivo de impedir el acceso a los jóvenes que carecen de talento, la disposición o la voluntad para seguir los estudios superiores, reducir la tasa de 
deserción motivada por los bajos rendimientos académicos, ...”.

\section{DESARROLLO DE LA PAA-UCR}

1960: El sistema planteado fue aprobado por el Consejo Directivo de Ciencias y Letras y luego ratificado por el CU; las pruebas correspondientes se llevaron a cabo el 7 de enero de 1960. El sistema aprobado y aplicado consistió en:

1. La consideración de las calificaciones obtenidas en los exámenes de Bachillerato.

2. Una prueba de conocimientos que contenía 100 preguntas, de alternativa múltiple.

3. Una prueba de Aptitud General para estudios superiores, confeccionada sobre la base en las fórmulas experimentadas durante años por universidades norteamericanas.

4. Un examen de redacción, de no más de 2 páginas sobre un tema que debían escoger entre 5. El procedimiento de evaluación seguido fue el siguiente: cada candidato recibió una calificación en la que se tenían en cuenta los resultados de sus calificaciones de Bachillerato, la Prueba de Conocimientos y el Test de Habilidad General, todo ello debidamente ponderado. Luego se dio un rango de calificación de 1 al 1000 a cada candidato. El CU al igual que el Consejo Directivo de Ciencias y Letras consideró que este sistema no era perfecto pero si perfectible.

Una vez integrado todo el material de la prueba está fue administrada experimentalmente a 250 estudiantes de primer año de la facultad de Ciencias y Letras, con el fin de verificar la validez y confiabilidad de la misma y conocer el grado en que los resultados se relacionaban con los obtenidos en las pruebas de conocimientos. En el transcurso de ese año, se sometieron a análisis estadísticos los resultados de la prueba experimental con el objeto de determinar: el poder discriminativo, el nivel de dificultad y el Poder Predictivo.

Así las cosas, esta prueba fue creada en un proceso de retroalimentación con el "Scholastic Aptitute Test” (SAT); una prueba de aptitud académica que se ha venido desarrollando desde principios de siglo, cuando se inició este tipo de práctica de selección de estudiantes para la educación superior en los Estados Unidos, en el Educational Testing Service (ETS) en la Universidad de Princeton. En ese proceso participaron ampliamente tres personeros latinoamericanos: el Dr. Jorge J. Dieppa por la Universidad de Puerto Rico, donde surge el College Entrance Examination Board de Puerto Rico, hoy en día College Board PRAL (Para América Latina) en el año 1963 y acompañado por el Dr. Adolfo Fortier, su primer Director; la Dra. Beatriz Molina por la Universidad de Guatemala, (entrev. 10) una universidad privada que 
hoy en día es cliente del College Board PRAL (entrev. 23); y el Dr. Gonzalo Adis Castro por la UCR en la que se constituye el IIP y donde se desarrolla su propia PAA.

Se inicia el establecimiento del Centro de Investigaciones Socio-Pedagógicas, creado por el Consejo Universitario a fines de año, a solicitud de los integrantes del Comité de Evaluación y sobre la base de la labor realizada por ellos. Dicho centro mantendría la dirección de los Exámenes de Admisión de primer año y debía dar concurso en los procesos evaluativos de las diferentes escuelas, departamentos, carreras y currículo. También, tenía que hacerle frente a la situación de los estudiantes superdotados.

Entre las funciones iniciales del Centro estaban llevar a cabo investigaciones en el campo de la Psicología, para la consulta y asesoramiento de los profesores que trabajaban en ese campo. Además se le encomendaba construir las pruebas de admisión y otros test para el Sistema de Admisión que se perfilaba (Sistema de Admisión, 1979). La labor del Sr. Adis Castro era crucial en actividades como investigación, el planteamiento de algunas monografías para las escuelas de Ingeniería, Derecho y Farmacia, el folleto “Cómo tomar apuntes”, "Hábitos de higiene”, entre otros (UCR Anales: 1960).

Ya en 1960, es un hecho, el examen de admisión se había hecho extensivo a todas las facultades de la Universidad y en todas sus sedes, aún así el Consejo Universitario da su autorización para que diferentes escuelas realicen pruebas específicas, adicionales a la prueba general. Así las cosas, el 19 de enero de 1960 el CU acuerda autorizar a la Facultad de Educación para que aplicara pruebas de admisión sobre las asignaturas de Matemática y Castellano a grupo de candidatos a estudios docentes de primaria ( C.U. Sesión 1050-03 del 19 de enero de 1961); sin embargo, en una sesión posterior un año después, se la autorizo a realizar exámenes a todos (as) los(as) estudiantes que desearan ingresar a ella, siempre y cuando hubieran aprobado el Examen de Admisión General (C.U., Sesión 1116-23 del 18- de enero de 1961).

1961: Para este año, a partir del 29 de mayo de 1961, se dejó de ser el Centro de Investigaciones Socio-Pedagógicas para llamarse Centro de Investigaciones Psicológicas. Según lo expuesto por el Dr. Adis, en el informe del Centro de Investigaciones Psicológicas (UCR, 1961), el cambio de nombre buscaba ampliar la actividad de dicho centro, con el fin de que se realizan a futuro investigaciones psicológicas, sociales y pedagógicas en relación con la realidad de la población estudiantil. 
El Examen de Admisión es la prioridad principal del centro, para esto se realizaban análisis de los ítems en maquinas de la IBM, y para conseguir nuevas preguntas, anteriormente, solicitaban a los profesores de tiempo completo de la Facultad de Ciencias y Letras que las formularan, como nuevo procedimiento contrataron a 16 Profesores de Enseñanza Media para que elaboraran las preguntas. El examen constaba de dos formulas, A (Estudios Sociales, Castellano) y B (Biología, Química, Matemática y Física (UCR Anales: 1961).

1962: El 29 de enero de 1962, el CU analiza el informe de la Comisión de Planes de Estudio y Programas y acuerda: exigir a los estudiantes de Escuelas Normales que aspiren a ingresar al II año de la Facultad de Educación presentar el examen general de admisión a la UCR; éstos deberán aprobar estudios generales antes de matricular II año. (C.U., Sesión 1191-13 del 29 de Enero de 1962).

1967: Diez años después del III Congreso, en 1967, el IV Congreso Universitario se enfrenta al problema de la admisión diferida y se ocupa nuevamente de replantear políticas de admisión (C.U. Correspondencia octubre 1991).

1969: Se cambia el nombre de Centro de Investigaciones Psicológicas por Instituto de Investigaciones Psicológicas (IIP) (C.U. Sistema de Admisión a la UCR, “1991”), nombre que conserva actualmente.

1970: En la sesión 1799-01, se acuerda que los graduados de la Escuela Normal Superior se admitirán en la UCR sin hacer examen de admisión ni los Estudios Generales (C.U. Sesión 179901, del 16 de noviembre de 1970).

1974: El proceso de admisión era definido por el CU. Se establecía una nota mínima de admisión (C.U. Sistema de Admisión a la UCR, “1991”).

1976: Se aprueba el primer Reglamento del Examen de Admisión conocido (C.U. Sistema de Admisión a la UCR, “1991”). El primer reglamento de admisión conocido, se ocupa de regular la realización del examen, únicamente.

1977: El 21 de diciembre de 1977, en la sesión 2451 artículo 05, se autoriza al Rector para que hagan de conocimiento público los resultados del Examen de Admisión mediante cifras globales y por sedes (C.U., Sesión 2451-05 del 21 de diciembre de 1977).

1979: En noviembre de este año, se aprueba el Reglamento acerca de Política de Admisión y Sistema de Ingreso, con la aprobación de este nuevo reglamento se derogó el anterior (Sistema de Admisión a la UCR, “1991”) y estuvo vigente hasta el 2003. En él, se 
formula una declaración de principios y objetivos. Entre ellos se habla se brindar igualdad de oportunidades a todos los individuos capaces de seguir estudios universitarios, seleccionar a las personas que constituyen una mejor promesa académica y orientar y ayudar a los estudiantes para que logren un optimo rendimiento.

Esta era la situación hasta mayo de 1990, cuando el V congreso apunta a la excelencia académica. (C.U. Correspondencia octubre 1991).

1982-83: Alrededor de estos años se da un cambio técnico-tecnológico de gran relevancia para la Prueba, este fue el paso de la lectura y calificación de la prueba que originalmente siempre se dio en forma "manual", al estilo tradicional, a la lectora óptica.

Así lo expresó la Directora del IIP de entonces, "cuando se trajo la primera lectora óptica se tuvo que pedir permiso hasta al Departamento de Estado. En sus inicios se tenía que ir a Puerto Rico a leer las hojas de respuestas, había que ir y venir. Un año en que hubo un atraso tuve que ir a México, allí se descompuso la máquina, por lo que de ahí tuve que ir con todo el material hasta Minnesota; fue una locura pasar por los aeropuertos con esas cajas con material confidencial...; por suerte al año siguiente llegó la máquina y todo se volvió más fácil”.

También en esos años se da una gran preocupación por el tema de la calibración de la Prueba de año a año. Se quería controlar que el nivel de dificultad de la misma se mantuviera parecido a lo largo de los años. De modo que se inició ese proceso, ya vigente en el ETS en Princeton y el College Board PRAL, de garantizar que la prueba de un año tenga una dificultad parecida a la del año anterior.

Otro aspecto que estuvo sujeto a cambios, en ésta época, fue la forma de devolución de los resultados. El hecho es que los resultados se daban en enero de cada año, sin embargo, por la gran proliferación de universidades privadas, tuvieron que adelantarse a septiembre; entonces se ofrecían los resultados en las UCR, no obstante, esto se prestaba para que se surgieran conflictos, producto de quejas estudiantiles, motivo por el cual se comenzaron a enviar a los colegios.

Asimismo, se dan los primeros pasos en la atención a las personas con necesidades educativas especiales asociadas a discapacidad que comienzan a solicitar ingreso a la universidad.

Alguna otra variación se da con respecto al número de fórmulas que se aplican cada año. En general se han aplicado cuatro fórmulas simultáneamente, lo que ha prevalecido desde 
sus inicios hasta la fecha, no obstante, algunos años se aplicaron más o menos, dándose la particularidad de un año en que la Prueba se trató de doce fórmulas.

1985: En Junio de 1985 la Comisión de política de Docencia e Investigación, presenta el dictamen sobre la modificación al sistema de obtención de puntaje de admisión para primer ingreso y se acuerda: establecer la escala estándar de 200-800 con valores promedio 500 y desviación estándar 100, además como política experimental por dos años: dar instrucciones para desalentar las respuestas totalmente al azar y aplicar una fórmula que recompense la no respuesta con el mismo valor que el que se obtendría si esta se respondiera totalmente al azar, esto con el fin de aumentar el valor predictivo de la prueba ( C.U, Sesión 3195-05 del 27 de Junio de 1985).

En los años siguientes de discute este punto en forma reiterada. Luego se acuerda establecer como nota mínima para los dos próximos años: 442, que equivaldría al 60 en la escala 0 a100, y que va de acuerdo con conceptualizaciones que maneja el público del 60 como nota mínima de promoción en secundaria. ( C.U. Sesión 3413-16 del 14 de oct. 1987).

1988: Un año después, en Julio de 1988, en la sesión 3482, se “acuerda autorizar la presentación de Exámenes de Admisión en el extranjero, en el caso de que exista un número significativo de solicitudes y bajo control de un funcionario universitario, designado por el Rector de la Universidad de Costa Rica” (C.U Sesión 3482-08 del 19 de Junio de 1988).

1989: Se acuerda solicitar al IPP realizar una nueva evaluación del sistema de calificación que incluya al menos 2 años lectivos (C.U. Ses 3549-11, 28 de marzo de 1989).

1991: A partir de 1974 el proceso de admisión desarrollado había sido definido por el CU; éste decidía cada año el número de cupos disponibles en las diferentes sedes universitarias. Emerge la certeza de que hasta ese momento, el cupo había sido el factor determinante de ingreso y no el promedio o nota mínima de admisión como se creía y según se había venido estableciendo, como nota de corte, en $60 \%$ o 442. Se acuerda que sea el IIP el que defina y se haga responsable del aspecto técnico del proceso, mediante la elaboración, aplicación y calificación de la prueba. (Sistema de Adms. a la UCR, “1991”)

1993: En septiembre de 1993, el señor Rector informa que "IIP encontró una propuesta que satisface los dos intereses: uno es el interés de mantener la escala para las investigaciones y trabajos internos en 200-800 como es más conveniente, pero para efectos de publicar se pueda traducir a una escala de $\mathbf{0 - 1 0 0}$, de manera que sea posible anunciar que los estudiantes sacaron 
un noventa o un noventa y cinco, no como dicen ahora que sacó un 700”, lo que es difícil de conceptualizar para el público (C.U. Sesión 3978-05b del 28 de setiembre de 1993).

1995: El 14 de febrero de 1995 la Dra. Yolanda Rojas, Rectora a.i. rinde el informe referente a las cifras de solicitud de ingreso a la UCR correspondiente al periodo 1994-1995 (C.U. Sesión Ordinaria 4085-04e, 1995); informa que para ese período se inscribieron para realizar la Prueba de Admisión 16.200 estudiantes. De estos fueron elegibles con un promedio mayor a 442, 10.632. La Institución, de acuerdo con su capacidad, ofreció 4.788 cupos para estudiantes de nuevo ingreso”, concursaron 5.912 estudiantes de los cuales 1.770 escogieron opciones de carrera con una nota de admisión superior a la obtenida, por lo que para estos se va a ofrecer una segunda oportunidad con los cupos restantes (C.U. Sesión 4085-04e del 14 de febrero de 1995).

En marzo de 1995, en la sesión 4096-07, el CU conoce una propuesta para la aplicación de una PAA adicional durante el primer ciclo de 1995, para ingresar en el II ciclo lectivo de ese año (C.U. Sesión 4096-07 del 28 de marzo de 1995) que luego no procede. También se dan acuerdos de “apoyar gestiones tendientes a revisar y actualizar las PAA y declarar esta última como un proyecto prioritario de la Institución”, también se acuerda solicitarle al IIP “una propuesta de pruebas que permitan valorar las habilidades y destrezas de los estudiantes por áreas de interés, de acuerdo con el nuevo modelo de admisión. Dicha propuesta debe estar elaborada a más tardar el 31 de octubre de 1995.” brindando el apoyo financiero necesario y para que se ponga en ejecución en 1997 (C.U. Sesión 4116-04 del 14 de Junio de 1995).

1998: En octubre de 1998, la Comisión de Política Académica presenta el dictamen No CPADIC-98-34, relativo a eliminar el redondeo en el proceso de cálculo de la nota de admisión de la Universidad (C.U. Sesión 4393-06 del 14 de octubre de 1998), el proceso de análisis de dicho dictamen continúa en la sesión siguiente, en la cual se considera que "el cálculo de la nota de admisión en la 200-800 no utiliza decimales, lo cual limita la capacidad de discriminación”. Después del análisis del mencionado dictamen se acuerda: "solicitar a la Administración que en el cálculo de la nota de admisión se utilice un mayor número de cifras significativas, de modo que se garantice una mejor discriminación”, “ Que el puntaje de admisión se entregue con dos decimales”. ( C.U. Sesión 4394-05 del 20 de octubre de 1998).

1999: En 1999, el CU recibe una solicitud del diputado Sr. Walter Céspedes Salazar, tratada en la sesión 4431, en la que se pide “se considere la posibilidad de que la Institución prepare 
un examen de admisión especial para que los indígenas tengan la oportunidad de superarse personal y profesionalmente” (C.U. Sesión 4431-04a del 07 de abril de 1999).

2002: Se da recomendación del Equipo Técnico Investigador (ETI-PAA) para aplicar nota de corte variable fundamentada técnicamente. La "nota de corte” es el valor mínimo para tener la opción de ingreso, "elegibles"; pero realmente identifica al grupo que definitivamente no tiene opción de ingreso, esto sobre la base de que conforme baja el valor del resultado obtenido por el estudiante aumenta la expectativa negativa de no tener éxito en los estudios superiores que demandan las carreras universitarias.

Utilizar un puntaje de 442 como corte para definir a los examinados elegibles produce distorsión y confusión en el público, haciendo pensar a algunos que con un 442 en el puntaje de admisión hay posibilidades reales de ser admitido. Lo cierto es que, dado que la escala 200800 tiene un promedio de 500 y una desviación estándar de 100, normalmente habrá al menos un $60 \%$ de los examinados con una nota mayor o igual a 442.

Dado que se trata de un procedimiento de ingreso general a la Universidad, la selección se basa en la posición relativa que ocupa el desempeño de un individuo particular en relación con el desempeño de los demás que han concursado; es decir, que la interpretación se realiza siguiendo un modelo con referencia a normas (Gronlund \& Linn, 1990). En este tipo de modelos, los puntajes absolutos no se interpretan en términos de qué puede o no lograr el examinado en cuanto a ciertas tareas o contenidos específicos, como sí es usual en el caso de pruebas para evaluar conocimientos, cuando el modelo para la interpretación de los puntajes es referido a criterios. Siguiendo el modelo clásico, se seleccionan aquellos examinados que tengan los puntajes más altos, ordenados de mayor a menor, hasta llegar a llenar los cupos disponibles (Whitney, 1996). Así, en lugar de tener una nota de corte fija, ésta debe establecerse cada año, estimando del número de cupos disponibles. (ETI-PAA, archivos).

2003: Paso de la Teoría Clásica de los Tests a la Teoría de Respuesta a los Ítems. "La Teoría de Respuesta a los Itemes, TRI, (o “Item Response Theory”, en inglés) es un enfoque más poderoso que la Teoría Clásica de los Tests para el análisis de calidad técnica de instrumentos de medición psicométricos. Comparada con la Teoría Clásica, la TRI permite obtener mayor información sobre los ítemes de la prueba y sobre el constructo o variable latente que se pretende medir. Además, con la TRI se pueden generar pruebas adaptadas al nivel de los examinados. También se logran indicadores de las propiedades psicométricas del instrumento y de los ítemes que lo 
componen, según los diferentes valores del constructo de interés. Estas características de la TRI le dan ventajas esenciales en relación con la Teoría Clásica”. (Montero, E.: 2001).

2004: A inicios del 2004, el CU se refiere a la comunicación realizada por el IIP, referente a variaciones en la PAA, tales como la eliminación de la posibilidad “no responde" dentro de las opciones de respuesta. (C.U. Sesión 4862-03f del 24-02-04, gaceta: 04 del 15-03-04). De acuerdo con el oficio R-6957-2003 del Sr. Rector, se aprueba la eliminación de la Fórmula Correctiva del Azar para la calificación de la PAA a partir del 2004 y con ello la eliminación del "No Respondo" (NR) en las opciones de respuesta a los ítems (Archivos ETI-PAA). .

Previamente se había logrado evidenciar, por medio de una encuesta de salida del ETI a los examinados, que los supuestos en el uso de la fórmula, a esa fecha, eran de dudosa aceptación y no necesariamente garantizaban la equidad para los examinados. Los estudiantes no estaban haciendo el uso esperado del NR, muchos lo estaban utilizando como artificio para lograr puntos extra cuando no les daba tiempo para resolver el examen. Así, en adelante se calificará sencillamente el porcentaje de respuestas correctas.

Se decide contratar a una empresa, por medio de licitación pública, dedicada al desarrollo de sistemas de información, para la elaboración de un softwear especializado en el manejo de un “Banco de Ítems y Montaje Automatizado de Fórmulas Paralelas”. (IIP-ETIPAA-Centro de Investigaciones en Computación del Instituto Tecnológico de Costa Rica: 2004). Asimismo, se crean dos nuevas áreas en el ETI: Psicología y Educación Especial y se fortalece la de Estadística y Psicometría

2006: Se actualiza el estudio de “Análisis de Sensibilidad” o Función Diferencial de los Ítems (DIF). Este tipo de análisis se ha venido realizando siempre, desde los inicios de la prueba, en que se trata de construir ítems libres de contenidos o contextos que pueden afectar negativamente a ciertos grupos de examinados(as), esto quebrantaría los principios de estandarización, afectando su desempeño y por ende la confiabilidad y validez, así como la equidad que debe prevalecer en los procesos de selección justa.

"Se posibilita su reconocimiento en el contexto de una sociedad que pretende ser multicultural, multiétnica, no confesional, donde se respetan diferentes costumbres y estilos de vida” (Montero, E.: 2007). Así, se hace necesario tener en consideración aspectos que son familiares solamente a algún (os) segmentos de la población de examinados para descartarlos, tales como: conocimiento previo, familiaridad (semáforo, rotonda, viaje en avión, deportes), 
situaciones "delicadas" o que pueden evocar emociones negativas en la población o en un cierto grupo de población: dolor, enfermedad, miedo, enojo, ansiedad, temas religiosos o controversiales (aborto, matrimonio gay, TLC), estereotipos (la gente de raza negra es muy buena para el baile, los latinos son muy románticos), contextos que brindan un claro posicionamiento ideológico, contextos que puedan ser ofensivos para ciertos grupos culturales, étnicos, u otros

Contrariamente, se deben construir y/o reconstruir ítems que no presenten sesgo de género, como podría ser usar roles estereotipados, de contextos socioculturales o tipos de instituciones educativas o de grupos étnicos, entre otros; sino recurrir siempre a contextos que sean igualmente familiares para todos los examinados. (Montero, E.: 2007).

2007: Es realmente impresionante el aumento de solicitantes en los últimos diez años en que a partir del 97 se ha quintuplicado la población de examinados; en ese año con una capacidad total para albergar 5.447 estudiantes de nuevo ingreso, fueron procesas 6.641 solicitudes, de las cuales fueron aceptadas 4.749, dejando de esta forma 1.892 estudiantes fuera del sistema universitario. Para el proceso de admisión 2001-2002, el Sr. Rector informa que de "los 25.881 estudiantes que realizaron el examen, 16.300 obtuvieron nota mayor igual al corte de 442, es decir que eran elegibles, cuando el número de cupos era de 6.000, aproximadamente” (C.U. Sesión 4684-02b del 20 de noviembre del 2001). En el 2007, de alrededor de 35.000 aplicantes de la PAA se contó con la capacidad de aceptar aproximadamente 7.000 .

2008: Por primera vez se efectúa el montaje de la PAA utilizando el software especializado y construido para ello y a partir del Banco Automatizado de Ítems; esto con éxito en el área de matemáticas y alguna dificultad en la de verbal.

\section{CONCLUSIONES:}

La Prueba de Aptitud Académica (PAA) la podemos catalogar como un Test de selección de alto impacto, dado que ha sido construida y utilizada, a través de tantos años, para elegir los mejores candidatos a cursar carreras en la UCR, es decir, para la toma de decisiones de altas consecuencias para quienes la realizan, esto como requisito de ingreso a la UCR y para los objetivos y fines de una Educación Superior de calidad. Nace como un examen de admisión, que ha pasado por varias etapas en sus procedimientos, evidenciando necesidades de retroalimentación constante, tanto en la fundamentación teórica como en la creación constante de nuevos ítemes. 
Las necesidades y demandas que gestan esta prueba se empiezan a hacer evidentes desde el año 1942 hasta el 57 en que se toma la determinación de establecer un "Sistema de Admisión" propiamente dicho, se toman las determinaciones respectivas y dicho sistema empieza a regir en 1960 con la primera PAA-UCR propia. Responde a la gran demanda de estudios superiores de una población joven en incremento muy pronunciado, principalmente en los últimos 10 años, ante la que se hizo necesario, dados los serios problemas de deserción, seleccionar a los más aptos y responder a la demanda de elevar los niveles académicos y científicos que se dan, precisamente, a partir de ese momento en el ámbito internacional, lo que ha colocado a la UCR en una posición muy respetable.

Así las cosas, la UCR ha optado por utilizar instrumentos de selección de estudiantes como este con la intención de seleccionar a los estudiantes que tengan mayores posibilidades de alcanzar éxito académico; esto sobre la base de razones de tipo económico-político, en lo que "los estudios" se constituyen en una inversión tanto de la institución como del estudiante, en términos de recursos materiales y también de tiempo. Esta posición es aceptada por la sociedad académica nacional e internacional, en vista de que las aspiraciones y logros profesionales marcan un cambio cualitativo y vital en el proyecto de vida personal de los candidatos, adquieren sentido en la medida en que se constituyen en un aporte a la persona, y no en un obstáculo (meta inalcanzable) para su realización sino para canalizar logros en otras alternativas de superación personal que ofrece la sociedad. Se ha desarrollado un instrumento psicométrico de aptitud académica general que constantemente está siendo probado en términos de validez y confiabilidad, y generando criterios técnicos que le permitan seleccionar a sus candidatos a ingreso, la Prueba de Aptitud Académica (PAA).

Como es posible observar, el proceso para llegar al sistema actual de admisión ha sido extenso y a requerido del interés y el esfuerzo de muchos. También ligado a este proceso se ha dado otro de crecimiento de la población estudiantil, que año con año solicita la oportunidad de ingresar a la Universidad de Costa Rica y que requiere de una adecuada orientación vocacional en todo el Sistema Educativo a nivel Nacional.

A lo largo de su evolución, a la PAA se la ha concebido siempre como un test de aptitud que valora la potencialidad del estudiante para el desempeño académico a nivel universitario; diferentes análisis psicométricos han evidenciado que dicha capacidad se refleja en habilidades 
verbales y matemáticas. Se ha construido y reconstruido a través de los años sobre una premisa básica de equidad, buscando que no involucre discriminaciones a favor o en contra de determinados grupos de educandos, por su procedencia educativa, social o racial (González, A.: 1985), y sobre la base de comprobaciones realizadas con estudios de predictividad de rendimiento académico, lo que la ha caracterizado siempre y consolidado firmemente su permanencia.

Dadas sus grandes bondades en cuanto a indicadores de validez y confiabilidad y en la predicción de rendimiento académico, como requisito básico para su utilización como instrumento de selección e ingreso, ésta debe ir cambiando, de acuerdo a las necesidades presentes en la sociedad actual y en respuesta a la demanda, que se incrementa cada vez, de mejor y diferente educación, como lo ha hecho en el pasado.

Durante los últimos seis años y en lo que se proyecta para los venideros, lo que corresponde a lo que llevamos de la primera década del siglo XXI, se da una efervescencia de desarrollos y actualizaciones teóricas, técnicas y tecnológicas, que se palpan en determinaciones muy concretas producto de la aplicabilidad de avances científicos en el campo de trabajo, que realiza el IIP-ETI-PAA.

Finalmente se observa, que a través de los años, se ha acrecentado la confianza de las Autoridades Universitarias en el IIP-ETI-PAA, esto porque se ha ido depositando la responsabilidad directa de los aspectos técnicos en este grupo, que se ha distinguido por su gran profesionalismo y se constituye en personal de confianza de la Institución. Inicialmente era el Recto y autoridades colaboradoras quienes tomaban las decisiones más importantes acerca del proceso de selección, luego el Consejo Universitario fue asumiendo una buena parte y encontrando cada vez más apoyo en dicha entidad.

Todo ello nos compromete y llama, principalmente a la UCR pero a todo el Sistema Educativo Nacional, a una política muy clara y definida, no solo de cobertura sino también de calidad educativa, tan clara, certera y concreta como la PAA misma.

\section{BIBLIOGRAFÍA}

Estrada, R. “65 Años Creando Valor con Valores”, Líderes Mexicanos. 2008, Tomo 132.

Gámez Solano, U. “Informe del Secretario General”. Anales de la UCR 1952 y 1953.

Coronado, M.”Inf. del Departamento de Bienestar y Orientación”, Anales de la UCR. 1955.

Facio, Rodrigo.”Informe de la Rectoría”. Anales de la Universidad de Costa Rica. 1955.

Padilla, E. “Inf. Director del Departamento de Bienestar y Orientación”, Anales UCR. 1957 
Coronado, M. “Inf. Director del Dep. de Bienestar y Orientación”. Anales de la UCR. 1959. UCR. Anales de la Universidad de Costa Rica. 1953, 1954, 1956, 19591960 y 1961, UCR. Universidad de Costa Rica. Consejo Universitario. Archivos 